\title{
EPÓNIMOS PROCEDENTES DEL ALEMÁN DE LA ECONOMÍA, LA FÍSICA Y LA TÉCNICA
}

\author{
ALFONSO CORBACHO SÁNCHEZ \\ Universidad de Extremadura
}

\begin{abstract}
Resumen
Como sucede con los préstamos lingüísticos en general, los epónimos alemanes que han entrado en el español dan fe de algunos campos en los que los países de lengua alemana han influido en el mundo hispánico a través de la historia. Así, el elevado número de epónimos médicos de origen alemán que adopta el español a lo largo del siglo XX revela la importancia del desarrollo de la medicina y disciplinas afines en Alemania, Suiza y Austria durante ese período. Estos epónimos han sido estudiados con frecuencia por médicos y lingüistas, sobre todo lexicólogos; pero a otros, como los de los campos de la economía, la empresa, la física y ciertas especialidades de la ingeniería, se les ha prestado menor atención. El presente trabajo se concentra solamente en estos últimos; y más concretamente en su importancia en la enseñanza del alemán a hispanohablantes.

Palabras clave: epónimos, germanismos, préstamos alemanes y enseñanza del alemán.
\end{abstract}

\begin{abstract}
As happens with loanwords in general, the German eponyms borrowed by the Spanish language are evidence of some fields in which the German-speaking countries have influenced the Hispanic world throughout history. Thus, the large amount of medical eponyms of German origin that have entered Spanish during the twentieth century reveals the importance of the development of medicine and related disciplines in Germany, Switzerland and Austria during this period. These eponyms have frequently been dealt with by physicians and linguists, especially lexicologists; but much less attention has been paid to others, such as those from the fields of economy, business, physics or certain branches of engineering. This paper focuses only on the latter type of eponyms; and discusses their importance in the teaching of German to speakers of Spanish.
\end{abstract}

Key words: eponyms, germanisms, German loanwords and German language teaching.

\section{Introducción}

n cualquier estudio serio que intente profundizar en la naturaleza del léxico de una lengua no se puede soslayar la influencia de esos otros Jidiomas que, por unas u otras razones, han entrado en contacto con ella. En concreto, es inevitable prestar cierta atención a esos préstamos, calcos semánticos, etc. que a lo largo de la historia han enriquecido su tesoro léxico desde fuera. Ese 'tráfico' interlingüístico puede obedecer a razones muy diversas, pero los vínculos que establecen la proximidad o la vecindad en ningún caso pueden ser ajenos a este fenómeno. De ahí que, aunque el alemán no sea precisamente una de 
las lenguas que mayor huella haya dejado en el español, de vez en cuando nos topemos con términos procedentes de esa lengua que dan fe no sólo de una proximidad, al menos en Europa, sino de una relación continuada entre el mundo hispánico y el de lengua alemana, y viceversa. Por supuesto, esa interrelación difícilmente será comparable, sobre todo en cuanto a la cantidad, con la que se establece entre el español y el inglés, o el alemán y el inglés, dado el estatus de 'lingua franca' de que goza este último idioma hoy en día en numerosos campos del conocimiento y esferas profesionales. Sin embargo, sí lo es en cuanto a la calidad, sobre todo en ciertos campos profesionales y dominios de la ciencia en que tanto Alemania como los demás países de lengua alemana han alcanzado cotas como mínimo comparables a las del mundo anglosajón. La economía y la actividad empresarial, la técnica o la física, por no decir nada de la medicina, ofrecen muestras muy elocuentes en el sentido referido.

Con esta orientación y por razones de diversa índole -que no siempre precisan una aclaración pormenorizada- a las que se unen obvias preferencias personales, se ha escogido como motivo del presente trabajo un tipo específico de préstamo que suele darse con mayor frecuencia en los ámbitos científicos y profesionales antes mencionados. Se trata en concreto de una serie de epónimos procedentes del alemán que han tenido fortuna en el español, formando ya parte de nuestro acervo léxico especializado de esos ámbitos específicos.

Este tipo de préstamos -al igual que ocurre con los propios de la filosofía, el arte en cualquiera de sus manifestaciones, o cualquier otra parcela del saber o la ciencia- ponen de manifiesto el grado de desarrollo y la influencia que en un momento determinado ha tenido el país emisor. En este caso, nos hablan del liderazgo científico y tecnológico que han ejercido sobre todo Alemania, y también los demás países de lengua alemana, en algunas áreas y disciplinas desde al menos las primeras décadas del siglo XX. El trasvase de esa magnífica aportación -ya sea bajo la forma de inventos, nuevas teorías o leyes científicas- al contexto hispánico se ha traducido, en no pocas ocasiones, en epónimos de la antroponimia alemana que no sólo designan esos objetos o conceptos novedosos, sino que inmortalizan, también entre nosotros, a sus ilustres autores.

La medicina ${ }^{1}$ es con mucho el área de conocimiento que cuenta con el mayor número de formaciones eponímicas procedentes del alemán y, además, este léxico especializado es también el que mayor atención ha recibido. De ahí que en este trabajo apenas se tomen en consideración a esos epónimos concretos, y se dedique más espacio a los pertenecientes a los campos de la economía, la empresa, la física y la técnica, que, según se ha indicado más arriba, son menos y, en todo caso -a pesar de su indudable interés-, han recibido una atención menor. Sea como fuere,

1 Para apoyar nuestra afirmación, bastaría recordar aquí el catálogo más amplio de epónimos médicos que podemos encontrar en la red Who named it? The world's most comprehensive dictionary of medical eponyms y otros trabajos lexicográficos como son: Peñate y Baladrón (2000), Forbis et al. (1998), Pschyrembel (1998), Hombourger et al. (1968), Leiber/Olbert (1968), el Diccionario médico Roche (1993) y el Diccionario Terminológico de Ciencias Médicas (1998). 
se intentará, dentro de las limitaciones y la exigüidad de esta contribución, si no llenar esta laguna, proporcionar al menos una orientación general sobre la cuestión indicada.

Las fuentes del breve inventario que se ofrece a continuación son muy variadas, dentro de la lexicografía monolingüe general y específica (español y alemán) o bilingüe también general y específica (en este caso, no sólo diccionarios de español/alemán sino también de español/inglés), junto a otros trabajos de diversa índole entre los que, además, se incluyen una considerable nómina de páginas web. Los datos bibliográficos de todas las obras consultadas aparecen debidamente referidos al final de estas páginas.

Así pues, antes de entrar en la consideración de los epónimos, nos ocuparemos de la delimitación conceptual de germanismo con la que sistemáticamente también se identifican estos préstamos.

\section{Germanismos del alemán}

Se decía al principio que el epónimo procedente de otra lengua constituye un caso muy específico de ese fenómeno general que denominamos préstamo lingüístico. $Y$ en ese sentido no cabe duda de que la inmensa mayoría de los epónimos españoles procedentes de países de habla alemana se agrupan en la categoría de germanismos, término no demasiado fácil de delimitar, pero no por ello indelimitable. En efecto, el término, como es sabido, se ha usado tradicionalmente, para referirse a todas aquellas palabras o estructuras que penetran en el español procedentes del alemán. No obstante, resulta a todas luces improcedente asociar e identificar todo lo germánico ${ }^{2}$ única y exclusivamente con el alemán, puesto que, como nos recuerda García Yebra ${ }^{3}$ (1984: 383), "ese término podría aplicarse a las interferencias de las lenguas germánicas en general".

\footnotetext{
2 Todo apunta a que los préstamos germánicos más antiguos son los de procedencia visigótica que datan del siglo $\mathrm{V}$, dado que fueron los visigodos -junto a los vándalos, suevos y alanos- el pueblo germánico más numeroso y relevante de los que tomaron parte en la invasión de la Península Ibérica. Para más descripciones pormenorizadas sobre los elementos germánicos, véanse los estudios de Gamillscheg (1967) y Quirós Rodriguez (2003).

3 Téngase en cuenta que en la actualidad se hablan 12 lenguas germánicas (más de 865 millones de parlantes): inglés, alemán, holandés, sueco, afrikaans, danés, noruego, frisón, yídis, luxemburgués, islandés y faroés (Del Moral, 2002: 217). Con la salvedad del inglés, que -sin dejar de ser un germanismo- en materia de préstamos cuenta con una denominación propia (anglicismo) debido a su prestigio internacional, todas las lenguas restantes quedan identificadas como germanismos, con lo cual este término no puede restringirse única y exclusivamente a la lengua alemana. El motivo principal que pueda justificar la carencia de una terminología de mayor calado y precisión para designar todos los préstamos germánicos reside en su menor permeabilidad y adaptabilidad lingüística a la hora de incorporarse al español frente a otras lenguas más próximas al castellano (García Yebra, 2004: 384). Para Gamillscheg (1967: 79): “Los elementos germánicos que sobreviven en la lengua española están, en su menor parte, tomados directamente de un idioma germánico. En la mayoría de los casos estos elementos provienen o directamente del latín vulgar o de otra lengua románica, en primer lugar del francés". Asimismo, conviene precisar que la invasión
} 
Y ya que, según también el citado académico, el término 'alemanismo' no se utiliza, somos partidarios de precisar la procedencia de los préstamos y decantarnos por la denominación de germanismos del alemán ${ }^{4}$ para referirnos a la voz o construcción originada en alemán y usada en español. Obviamente, en virtud de todo lo expuesto, somos conscientes de que se continuará aplicando el concepto de germanismo a los préstamos de origen alemán, a pesar de tratarse de cualquier vocablo procedente de las lenguas germánicas (cfr. Lázaro Carreter, 1987: 210), con la excepción del inglés.

Quizá se revele más interesante acudir, por ejemplo, al Diccionario de lingüística de Dubois (1979: 313) que distingue los germanismos antiguos de los germanismos modernos y entre estos últimos cita los del alemán. En sus palabras:

\begin{abstract}
Se llama germanismo a todo préstamo de origen germánico. Suele emplearse principalmente para designar los préstamos germánicos antiguos, que se introdujeron en el latín tardío o en la época visigoda (rico, guardar, ataviar, parra, etc.), aunque pueden distinguirse los germanismos antiguos y los germanismos modernos (por ejemplo, los préstamos del alemán: sable, feldespato, níquel, etc.). Los préstamos ingleses reciben el nombre de anglicismos.
\end{abstract}

Naturalmente, no es nuestra intención aquí profundizar en la delicada y compleja cuestión de la distinción entre germanismos antiguos y modernos. No obstante, al aludir a los germanismos, debería quedar siempre claro a cuál de ellos nos estamos refiriendo. De ahí que insistamos en la denominación de germanismos del alemán para identificar a todos los préstamos procedentes del alemán, excluyendo a las demás lenguas germánicas; y, además, por lo que aquí nos concierne con la cuestión de los epónimos, a todo el léxico relativo al período del 'Hochdeutsch', es decir, lo que se entiende en la actualidad por alemán estándar. En fin, hasta aquí hemos visto cómo se definen los germanismos; veamos a continuación como se describen los epónimos.

\title{
3. Epónimos del alemán
}

Sin ningún género de dudas, se puede sostener que es el plano léxico el más abierto de una lengua a la hora de recibir influencias extranjeras. Y, en esta línea, uno de los ámbitos en los que más nos llama la atención la influencia germánica se

\footnotetext{
germánica en España, trasladándonos a la época visigoda, fue ciertamente escasa y ello fue óbice para que el léxico hubiera tenido repercusiones de mayor envergadura en el español.

${ }^{4}$ Conviene precisar que en este estudio nos limitaremos únicamente al léxico perteneciente al período que se entiende como alemán estándar ('Hochdeutsch'), es decir, la lengua alemana desde finales del siglo XIX, justo cuando se alcanza un consenso en torno a la ortografía y la pronunciación. Por tanto, no entraremos en la división diacrónica de los períodos históricolingüísticos ('Althochdeutsch', 'Mittelhochdeutsch', 'Frühneuhochdeutsch' y 'Neuhochdeutsch') que conforman el 'Hochdeutsch' hasta que éste llegó a convertirse en el alemán estándar. Para una información más exhaustiva sobre la historia de la lengua alemana, véanse, entre otros, los manuales de Schmidt (1986) y Wolf (1986).
} 
refiere a la antroponimia. En efecto, el abanico de nombres propios de origen germánico -dicho sea de paso, que suelen estar compuestos por un sintagma bitemático- es ciertamente amplio. Valgan como botones de muestra los que siguen: Alberto (Albert 'noble brillante'), Alfredo (Alfred 'todo paz') o Luis/Ludovico (Ludwig 'ilustre en el combate') entre muchos otros (cfr. Quirós Rodríguez, 2003: 253).

Sin embargo, pasando a un plano más concreto, lo que nos proponemos en esta sección es tratar de dilucidar el concepto de epónimo ${ }^{5}$ y para este cometido nos hemos apoyado especialmente en algunos diccionarios de lingüística.

Cerdá Massó (1986: 100) es uno de los primeros en recogerlo y lo define escuetamente como "nombre patronímico que designa un pueblo, tribu, ciudad, época, etc.". En opinión de Cardona (1991: 98), que hace lo propio en su Diccionario de lingüística, un epónimo es un "sustantivo o adjetivo que se refiere a la transposición de un nombre propio de una persona a una cosa". Lázaro Carreter (1987: 166), estudioso de reconocido prestigio, también comenta el fenómeno y lo incluye en el Diccionario de términos filológicos, aunque no va mucho más allá en su definición al decir que se trata de un "héroe, persona, personaje literario, etc., que dan nombre a un pueblo, un lugar o una época" como la última edición del Diccionario de la Real Academia de la Lengua Española (2001) que, sobre la base mencionada, extiende su definición al campo de la medicina y de las unidades. En último lugar, reproducimos la que figura en el Diccionario de lingüística moderna de Alcaraz Varó y Martínez Linares (1997: 203) que se antoja un tanto más específica. El texto advierte que:

...se llama "epónimo" al héroe o a la persona que da nombre a un pueblo, a una tribu, a una ciudad o a un período o época. Por extensión, son "epónimos" los nombres comunes nacidos de un nombre propio, como un ford (por un coche de esa marca), una gilette (por una hoja de afeitar), etc.

Llegado a este punto, no parece que la cuestión conceptual afrontada en estas líneas esté definitivamente acotada. No obstante, se utilizará en esta contribución la voz de epónimo como término genérico para referirse a cualquier palabra "derivada de un nombre propio o idéntica a él" (Newmark, 1995: 268) que aluda a un fenómeno u objeto enmarcado, por lo que aquí nos compete, en los campos de la economía, la empresa, la física y la técnica y que suele llevar, por lo general, el nombre del inventor o descubridor del fenómeno u objeto por él descubierto. Es obvio que en estos casos, en los que se asocia el significado al nombre de una persona, el nombre propio alcanza tal grado de generalidad que se convierte en un auténtico nombre común. El estudioso Newmark (1995: 268) diferencia, sin embargo, tres tipos de epónimos: los que derivan de nombres de personas, objetos

\footnotetext{
${ }^{5}$ Quizá convendría empezar constatando que la palabra epónimo proviene del griego ('epi' = encima, sobre) y ('onomos' = nombre), siendo su significado etimológico 'sobre el nombre'. Al hilo de esta afirmación, también sería digno destacar la tendencia a deificar a los héroes que reinaban en la Grecia clásica como, por ejemplo, al dar el nombre de Alejandría en honor a Alejandro Magno.
} 
y lugares. Serán los epónimos derivados de nombres de personas a los que dedicaremos nuestra atención en las páginas que siguen.

Tras estas breves calas en torno al concepto de epónimo, debemos pasar a la exposición de los materiales léxicos que sustentan los resultados de este trabajo, insistiendo una vez más en que la recopilación de los materiales se ha realizado, fundamentalmente, desde un punto de vista sincrónico. Por ello, la selección aquí presentada pretende ser claramente indicativa, dejando el camino abierto a otras inquisiciones que la completen por medio de estudios con un enfoque más amplio. Insistiremos, además, en que la relación mostrada no aspira a ser, desde luego, ni exhaustiva ni amplia por la imposibilidad de incluir aquellas subdisciplinas que pueden o no ser catalogadas como propias del mundo de la economía, la empresa y la técnica; amén de, como se ha indicado más arriba, la exigua influencia del alemán sobre el español.

Teniendo en cuenta las observaciones anteriores, procedemos a continuación a la transcripción de los materiales localizados por orden alfabético, siguiendo fielmente la ortografía original de los epónimos:

'Baedeker' (<Karl Baedeker). Marca comercial de guías turísticas que deben su nombre a su fundador, el editor alemán Karl Baedeker (1801-1859). El término 'Baedeker' alcanzó tanta fama que se ha convertido en sinónimo de guía de viaje.

'Bosch' (<Robert Bosch). El ingeniero alemán Robert Bosch (1861-1942) fue pionero en la construcción de accesorios eléctricos para la industria del automóvil y precursor de la compañía 'Bosch $\mathrm{GmbH}^{\prime}$. Asimismo, inventó las bujías que llevan su nombre.

'Boss' (<Hugo Boss). Empresa, inicialmente, diseñadora de prendas de vestir que, años más tarde, conseguiría reconocimiento mundial debido a sus fragancias. Fue creada en Metzingen por el alemán Hugo Boss a principios del siglo XX.

'mechero de Bunsen' (<Robert Wilhelm Bunsen). El químico y físico alemán Bunsen (1811-1899) fue quien popularizó este invento que lleva su nombre: un quemador o mechero de gas con entrada de aire y calor regulables utilizado en los laboratorios.

'Clausius' (<Rudolf Clausius). Físico alemán (1822-1888) que da nombre a la unidad de entropía, noción esta última que el mismo introdujo.

'Daimler' (<Gottlieb Daimler). El ingeniero alemán Daimler (1834-1900) desarrolló, junto a Maybach, el motor de combustión interna. Se le considera el padre de la marca Mercedes-Benz, teniendo en cuenta que la sociedad de motores Daimler acabaría asociándose con la de Karl Benz ${ }^{6}$ ('Daimler-Benz AG’).

'motor Diesel'7 (<Rudolf Diesel). Motor de combustión interna que recibió el nombre de su inventor, el ingeniero alemán Rudolf Diesel (1858-1913).

\footnotetext{
${ }^{6}$ Consúltese más adelante la entrada 'Mercedes'.

7 Para un tratamiento detallado de esta formación eponímica, véase Alvar Ezquerra (1996) que ya nos indicaba en su trabajo que el término ortográficamente correcto debía ser 'diésel' con el fin
} 
'Fahrenheit' (<Daniel Gabriel Fahrenheit). El físico alemán Fahrenheit (16861736) concibió una escala termométrica que conserva su nombre.

'Gauss' (<Carl Friedrich Gauss). Unidad de inducción magnética que recibe el nombre de Gauss (1777-1855), científico alemán conocido por sus importantes estudios en el campo de la física, en especial por sus trabajos sobre electromagnetismo.

'contador Geiger' (<Hans Geiger). El físico alemán Hans Geiger (1882-1945) inventó el 'contador Geiger', aparato que sirve para detectar y medir las partículas radioactivas.

'Hertz' (<Heinrich Rudolf Hertz). La unidad de frecuencia de vibraciones debe su nombre al físico alemán Heinrich Hertz (1857-1894), inventor del telégrafo y la radio sin cables.

'Lüger' (<George Lüger). Nombre comercial de armas cortas semiautomáticas inventadas por el austriaco George Lüger a finales del siglo XIX.

'Mach' o 'número de Mach' (<Ernst Mach). Unidad de medida que expresa la relación entre la velocidad de un cuerpo y la del sonido. Esta denominación es debida al apellido del filósofo y físico austriaco Ernst Mach (1838-1916).

'Maggi' (<Johannes Julius Maggi). Fábrica de productos alimenticios, conocida sobre todo por sus cubitos de caldo concentrado que deben su nombre al suizo Julius Maggi (1846-1912).

'Mauser' (<Wilhelm Mauser/Peter Mauser). Fusil de repetición alemán construido por los hermanos armeros Wilhelm Mauser (1834-1882) y Peter Mauser (1838-1914).

'Mercedes'. Nombre comercial de automóviles fabricados por la empresa alemana Daimler. El origen del nombre de la citada empresa automovilística data del año 1899. A la sazón, Jellinek, cónsul de Austria, ganó un rally con su vehículo Daimler bajo el nombre de Mercedes en honor a su hija. Unos años más tarde se sustituyó el nombre de la marca Daimler por el de Mercedes y se registró como marca oficial.

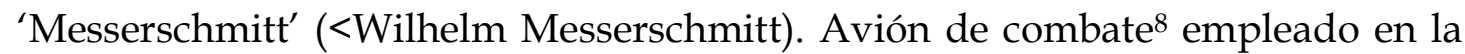
Segunda Guerra Mundial, cuyo diseño y nombre corresponde al ingeniero aeronáutico alemán Wilhelm Messerschmitt (1898-1978).

'Nestlé' (<Heinrich Nestle). Empresa mundial que pertenece al ramo de la alimentación, aunque en un principio se dedicara a la elaboración de productos farmacéuticos. Su nombre es una adaptación del apellido de su fundador, el químico germano-suizo Heinrich Nestle (1814-1890).

'Dr. Oetker' (<August Oetker). En 1891 el alemán August Oetker desarrolló la levadura en polvo ('Backin'). Así se fundó una de las empresas más importantes de

de que la ortografía fuera coherente con la pronunciación, tal y como acabó incorporándolo el DRAE (2001) en su última edición.

${ }^{8}$ Concretamente, se refiere al caza Messerschmitt 109, uno de los modelos que más se fabricaron durante el conflicto mundial. 
alimentación no sólo en Europa sino a escala internacional, creando la marca Dr. Oetker.

'Ohm' (<Georg Simon Ohm). Unidad de resistencia eléctrica que lleva dicho nombre en honor al físico alemán Georg Simon Ohm (1787-1854). Asimismo, descubrió la ley fundamental de las corrientes eléctricas: 'ley de Ohm'.

'Opel' (<Adam Opel). Empresa alemana fundada en 1862 por Adam Opel (18371895) que inicialmente se dedicó a la fabricación de máquinas de coser y bicicletas.

'motor Otto' (<Nikolaus August Otto). Motor de cuatro tiempos construido por el alemán August Otto (1832-1891), cuyo nombre se hizo genérico para este motor.

'Porsche' (<Ferdinand Porsche). Empresa alemana fabricante de automóviles deportivos. Su nombre proviene del apellido de su fundador, el ingeniero austriaco Ferdinand Porsche (1875-1951).

'Ritter-Sport' (<Alfred Ritter). En 1912 Alfred Ritter y su esposa Clara fundaron la fábrica de chocolate que lleva su apellido?.

'Siemens' (<Werner von Siemens). Alude, por un lado, a la unidad de medida de la conductancia eléctrica $y$, por otro, a la empresa alemana fabricante de material eléctrico y electrónico. Ambas recibieron su nombre en honor a su fundador, el ingeniero alemán Werner von Siemens (1816-1892).

'Strass' (<Joseph Strasser). El austriaco Joseph Strasser inventó en 1758 un cristal muy denso e incoloro, utilizado para imitar piedras preciosas y denominado 'Strass' en honor a su apellido.

'Weber' (<Wilhelm Eduard Weber). Unidad de flujo magnético. Su nombre recuerda al físico alemán Weber (1804-1891).

'Zeppelin' (<Ferdinand conde de Zeppelin). Globo dirigible, de tipo rígido, propulsado por motores que fue utilizado durante la Primera Guerra Mundial. Tomó su nombre de su diseñador, el industrial alemán Ferdinand, conde de Zeppelín (1838-1917).

En el apartado siguiente vamos a fijarnos más detenidamente en otras expresiones eponímicas que, si se nos permite, presentan ciertas 'anomalías' en su formación.

\subsection{Otras formaciones eponímicas}

Una lengua, a medida que van surgiendo nuevos referentes extralingüísticos, comienza a ampliar su capacidad designativa y para ello recurre, en algunos casos, a la creación de neologismos a partir de la combinación de varias letras, grupos de letras o sílabas, independientemente de que deriven o no de un nombre propio. Dicho esto, un repertorio de estas características también debe registrar aquellas otras voces eponímicas que son fruto de la abreviación, es decir, vocablos que se

\footnotetext{
${ }^{9}$ Unos años más tarde comenzaron a fabricar un formato de tableta de chocolate cuadrado con el fin de que tuviera cabida en los bolsillos de vestimentas deportivas, motivo por el cual se terminó añadiendo el vocablo 'Sport'.
} 
han formado artificialmente como resultado de combinar las sílabas o letras iniciales con sílabas o letras finales de nombres y apellidos. Así lo confirman los ejemplos siguientes:

'Adidas' (<Adi -Adolf- y Dassler ${ }^{10}$ ). Compañía multinacional alemana de artículos deportivos.

'Eduscho' (<Eduard y Schopf). Nombre comercial de un tipo de café que debe su nombre a su fundador, el alemán Eduard Schopf (1893-1935).

'Hertie' (<Hermann y Tietz). Una de las primeras cadenas de grandes almacenes en Alemania. Fue creada por el alemán de origen judío, Hermann Tietz (1837-1907).

'Rowenta' (<Robert y Wenta -Weintraud-). Compañía de electrodomésticos que fue fundada en Offenbach am Main por Robert Weintraud a finales del siglo XIX.

La presencia de estas muestras es abundante; podría decirse incluso que para las compañías es, hoy por hoy, uno de los procedimientos más utilizados en la creación de marcas comerciales que han de comprobarse "desde un punto de vista lingüístico, publicitario y legal" (Crystal, 1994: 115). Así, se encuentran otras expresiones eponímicas en las que además se esconden topónimos, como ocurre con:

'Haribo' (<Hans, Riegel de Bonn). Conocida marca de ositos de gelatina, cuyo nombre se compone del nombre y el lugar de nacimiento del fundador de la compañía, Hans Riegel (1893-1920).

'Ergee' (<Edwin Rößler de Gelenau -situado en el 'Erzgebirge'11_). Empresa alemana de calcetines y medias que fue fundada por Edwin Rössler en 1901.

Aunque no todo se reduce únicamente a nombres propios, además cabe la posibilidad de incluir junto a éstos sílabas que provienen de nombres comunes. Y el resultado provoca, por ejemplo, la designación de:

'Trigema' (<'Trikotwarenfabrik', 'Gebrüder' y Mayer). Nombre comercial de la empresa alemana fabricante de ropa deportiva. Fue fundada por los hermanos Mayer en 1919.

También pasaremos revista, siguiendo las coordenadas de los enunciados anteriores, a otras muestras de formación más arbitraria que utilizan para este cometido no sólo nombres comunes del alemán sino también vocablos procedentes del inglés. En este apartado se incluyen:

\footnotetext{
10 Adolf Dassler fundó la firma 'Adidas' en 1949 tras disolver la sociedad que tenía establecida con su hermano Rudolf, quien más tarde puso en marcha otro de los grandes fabricantes mundiales del sector deportivo (Puma).

${ }^{11}$ Cadena montañosa que se ubica en la frontera de Alemania y la República Checa.
} 
'Aldi' (<Albrecht y Discount -ing. descuento-). Cadena de supermercados.

'Chio-Chips'12 (<Carl, Heinrich e Irmgard von Opel). Empresa alemana de patatas fritas creada en 1962.

'Leica' (< Leitz $^{13}$ y Camera -ing. cámara-). Casa alemana dedicada fundamentalmente a la producción de cámaras fotográficas.

E incluso de otras lenguas como el italiano se ven afectadas por este proceso. Curiosa resulta, en este sentido, la muestra:

'Toblerone' (<Jean Tobler y 'torrone' -it. turrón-). Conocida marca de chocolate suizo que debe su nombre a Jean Tobler, quien emprendió su negocio en Berna en el año 1867.

Y ya para terminar con el asunto que nos ocupa, podemos dar fe, según se indicó anteriormente, que no son menos abundantes los ejemplos procedentes de la medicina ${ }^{14}$, pues son innumerables las enfermedades y los aparatos conocidos con el nombre de su descubridor o inventor como también son innumerables las ocasiones en las que los epónimos figuran en una amplia variedad de textos y hacemos uso de ellos sistemáticamente, ignorando, sin embargo, que ocultan un nombre y un hallazgo científico. Sirvan los ejemplos de: 'enfermedad de Alzheimer', 'angina de Prinzmetal', 'anomalía de Ebstein', 'síndrome de Asperger', 'enfermedad de Schönlein-Henoch', 'granulomatosis de Wegener', 'hernia de Bochdaleck', 'síndrome de Gardner-Silengo-Wachtel', 'síndrome de Reiter', etc.

Con estos pocos ejemplos, entre otros posibles e igualmente ilustrativos, hemos intentado acercarnos al conocimiento de los epónimos del alemán. Un listado de esta naturaleza revela mucho más que un simple nombre propio y sí todo un amplio abanico de testimonios culturales, históricos, sociológicos y científicos: una identidad, en tanto en cuanto el hombre está reflejado en ellos.

\section{Conclusiones}

De la muestra y los comentarios precedentes se desprende que, al menos en los últimos cien años, los principales préstamos del alemán al español -y por ende los epónimos más significativos- siguen perteneciendo a los campos de la ciencia y de la técnica. En concreto, los aquí tratados son indicadores fehacientes del gran desarrollo científico-técnico de los países de lengua alemana durante los siglos XIX

\footnotetext{
12 En este caso se da la particularidad de que se ha introducido un guión de separación entre el término que se ha construido sobre los nombres propios 'Chio' y la voz inglesa 'Chips'.

${ }^{13}$ A pesar de ser Leitz el nombre de la empresa, el invento corresponde a Oskar Barnack (Doval, 1996).

${ }^{14}$ No trataremos con mayor detalle, como ya se ha indicado, el asunto de los epónimos médicos, pues rebasaría con creces el marco que nos hemos propuesto en este trabajo.
} 
y XX y del enorme auge económico-empresarial que han experimentado algunos de esos países, sobre todo Alemania. En ese sentido, los préstamos eponímicos se revelan como auténticos 'embajadores' en el mundo hispánico de la sociedad y la cultura recientes de los países de lengua alemana; y, en la medida en que la enseñanza y el aprendizaje de una lengua extranjera no puede separarse del medio cultural y la civilización en que ésta se configura, esos préstamos tan singulares son también instrumentos didácticos de considerable importancia. Son, en efecto, un elemento motivador que atesora el español no sólo para el aprendiz del alemán como lengua extranjera para fines generales sino incluso para el economista, el empresario, el físico o el ingeniero que aprenden el alemán especializado de su área de conocimiento.

Además, nos permite llegar a la consideración de reconocer a los epónimos como términos con entidad propia junto al léxico patrimonial de cada uno de los campos de conocimiento mencionados en este trabajo. Como puede verse fácilmente, las denominaciones eponímicas han adquirido carta de naturaleza propia en el español por el hecho de que buena parte de ellas ya figuran registradas en los diccionarios.

Para concluir, tratando de adecuar las afirmaciones a las limitaciones de un trabajo de esta naturaleza, resulta innegable que, a pesar de su indudable importancia, el asunto de los epónimos procedentes del alemán aún constituye una pequeña laguna en el panorama investigador sobre la influencia de las lenguas extranjeras en el español. Como ya advertíamos al principio de estas páginas, creemos que los caminos de este fenómeno han sido escasamente abordados por la lexicografía, si nos atenemos a la profusa cantidad de publicaciones centradas en los epónimos en medicina.

\section{BIBLIOGRAFÍA}

\section{Manuales, diccionarios y artículos}

AA.DD. (1993): Diccionario médico Roche, Barcelona, Doyma.

AA.DD. (1998): Diccionario terminológico de ciencias médicas, Barcelona, Masson. $13^{\mathrm{a}}$ ed.

AA.DD. (1998): Pschyrembel Klinisches Wörterbuch, Berlin, Walter de Gruyter.

ALCARAZ ARIZA, M. A. (2002): “Los epónimos en medicina”, Ibérica, 4: 55-73.

ALCARAZ VARÓ, E. y MARTÍNEZ LINARES, M. A. (1997): Diccionario de lingüística moderna, Barcelona, Ariel.

ALVAR EZQUERRA, M. (1996): “Diesel”, Español Actual, 66: 79-81.

ALVAR, M. et al. (dirs.) (1967): Enciclopedia Lingüistica Hispana. Elementos constitutivos y fuentes II, Madrid, Consejo Superior de Investigaciones Científicas.

ALZUGARAY AGUIRRE, JJ. (1985): Diccionario de extranjerismos, Madrid, Dossat.

BEIGBEDER ATIENZA, F. (1988): Nuevo diccionario politécnico de las lenguas española e inglesa (2 vols.), Madrid: Díaz de Santos.

CARDONA, G. R. (1991): Diccionario de lingüística, Barcelona, Ariel. 
CERDA MASSO, R. (coord.) et al. (1986): Diccionario de lingüística, Madrid, Anaya.

COROMINAS, J. y PASCUAL, J. A. (1984): Diccionario crítico y etimológico castellano e hispánico, Madrid, Gredos. $1^{\text {a }}$ reimpr.

CRYSTAL, D. (1994): Enciclopedia del lenguaje de la Universidad de Cambridge, Madrid, Ediciones Taurus. (Ed. esp. dirigida por Juan Carlos Moreno Cabrera)

DEL MORAL AGUILERA, R. (2002): Diccionario Espasa: Lenguas del Mundo, Madrid, Espasa Calpe.

DOVAL, G. (1996): Diccionario de expresiones extranjeras, Madrid, Ediciones del Prado.

DROSDOWSKI, G. et al. (eds.) (2001): Duden. Deutsches Universalwörterbuch A-Z, Mannheim, Dudenverlag. $4^{\text {a }}$ ed.

DUBOIS, J. et al. (1979): Diccionario de lingüística, Madrid, Alianza Editorial.

FORBIS, P. et al. (1998): Stedman's Medical Eponyms, Philadelphia, Lippincott Williams \& Wilkins.

GAMILLSCHEG, E. (1967): "Germanismos", en Alvar, M. et al. (dirs.) (1967): 79-91.

GARCÍA YEBRA, V. (1984): Teoría y práctica de la traducción, Madrid, Gredos. $2^{\mathrm{a}}$ ed.

HERMANN, A. (1987): Lexikon. Geschichte der Physik A-Z, Köln, Aulis. $3^{\mathrm{a}}$ ed.

HOMBOURGER, P. et al. (1968): Enfermedades y sindromes con nombres propios. Diccionarios de epónimos clínicos, Barcelona, Laboratorio Dr. Esteve.

JÄGER, K. (ed.) (1996): Lexikon der Elektrotechniker, Berlin, VDE.

JERRARD, H.G. y MCNEILL, D.B. (1994): Wörterbuch wissenschaftlicher Einheiten, Wiesbaden, Quelle \& Meyer.

LÁZARO CARRETER, F. (1987): Diccionario de términos filológicos, Madrid, Gredos. $3^{\mathrm{a}}$ ed. $7^{\mathrm{a}}$ reimpr.

LEIBER, B. y OLBERT, T. (1968): Die klinischen Eponyme. Medizinische Eigennamenbegriffe in Klinik und Praxis, München, Urban/Schwarzenberg.

MOLINER, M. (1999): Diccionario de uso del español, Madrid, Gredos. $2^{\mathrm{a}}$ ed. $2^{\mathrm{a}}$ reimpr.

NEWMARK, P. (1995): Manual de traducción, Madrid, Cátedra.

PEÑATE, M. y BALADRÓN, J. (2000): Epónimos. Síndromes y Enfermedades con Nombre Propio en el examen MIR, Oviedo, Curso Intensivo Mir Asturias, S. L.

QUIRÓS RODRÍGUEZ, M. A. (2003): "Romania/Germania", Filología y Lingüística, XXIX (1): 241-259.

REAL ACADEMIA ESPAÑOLA. (2001): Diccionario de la lengua española, Madrid, Espasa Calpe. $22^{\mathrm{a}}$ ed.

SACKLOWSKI, A. y DRATH, P. (1986): Einheitenlexikon, Berlin/Köln, Beuth.

SCHMIDT, W. et al. (2007): Geschichte der deutschen Sprache. Ein Lehrbuch für das germanistische Studium, Stuttgart, Hirzel. $10^{\mathrm{a}}$ ed.

SECO, M. et al. (1999): Diccionario del español actual, Madrid, Aguilar. $2^{\mathrm{a}}$ reimpr.

SEIDEL, W. (2006): Woher kommt das schwarze Schaf? Was hinter unseren Wörtern steckt, München, Deutscher Taschenbuch Verlag.

SLABY, R. J., Grossmann, R., e Illig, C. (2001), Diccionario de las lenguas española y alemana (II tomos), Wiesbaden, Brandstetter. $5^{\mathrm{a}}$ ed.

VOLKMANN, P. (1994): Technikpioniere; Namensgeber von Einheiten physikalischer Größen, Berlin, VDE.

WAHRIG, G. (ed.) (2000), Deutsches Wörterbuch, Gütersloh, Bertelsmann.

WARNER, A. (2003): "Familiennamen deutscher Forscher für physikalisch-technische Maßeinheiten", Muttersprache, 4: 341-358. 
WOLFF, G. (2004): Deutsche Sprachgeschichte. Von den Anfängen bis zur Gegenwart, Tübingen, Francke. $5^{\mathrm{a}}$ ed.

WUBING, H.-L. (1992): Fachlexikon abc. Forscher und Erfinder, Thun/Frankfurt am Main, Harri Deutsch.

\section{Nómina de fuentes electrónicas}

Who named it? The world's most comprehensive dictionary of medical eponyms. http:/ / www.whonamedit.com (Consultado en octubre de 2007)

http:/ / de.wikipedia.org (Consultado en octubre de 2007)

http:/ / es.wikipedia.org (Consultado en octubre de 2007)

http:/ / www.adidas.com (Consultado en octubre de 2007)

http:/ / www.chio.de (Consultado en octubre de 2007)

http:/ / www.daimlerchrysler.es (Consultado en octubre de 2007)

http:/ / www.ergee.com (Consultado en octubre de 2007)

http:/ / www.haribo.com (Consultado en octubre de 2007)

http:/ / www.maggi.es (Consultado en octubre de 2007)

http:/ / www.oetker.es (Consultado en octubre de 2007)

http:/ / www.opel.es (Consultado en octubre de 2007)

http:/ / www.ritter-sport.de (Consultado en octubre de 2007)

http:/ / www.rowenta.com (Consultado en octubre de 2007)

http:/ / www.toblerone.ch (Consultado en octubre de 2007)

http:// www.trigema.de (Consultado en octubre de 2007) 